\title{
The Effect of Porous Structure on the Li Insertion/extraction Behavior of Carbon Gel Microspheres Encasing Silicon Powder
}

\author{
Izumi Yamada * and Shin R. MukaI \\ Graduate School of Engineering, Hokkaido University (Sapporo 060-8628, Japan) \\ Received November 29, 2009 ; Accepted January 19, 2010

\begin{abstract}
Silicon powder was encased within carbon gel microspheres with different porosities by simply adding silicon powder to the water phase during the inverse emulsion polymerization of resorcinol with formaldehyde, followed by drying and carbonization. From the results of charge-discharge experiments, it was confirmed that the reversible capacity of silicon and its cycle ability could be improved through such encasement and that the degree of
\end{abstract} \\ improvement depended on the porosity of the carbon gel matrix.
}

Key Words : Si Alloy, Sol-gel, Lithium-ion Battery

\section{Introduction}

Li-ion batteries have been widely used in portable electronic devices due to their high energy densities. Recently, Li-ion batteries have become to be regarded as a promising power source for electric vehicles (EV) and hybrid electric vehicles (HEV). Needless to say, batteries with higher capacities are required in such advanced applications. The negative electrode material commonly used in Li-ion batteries is graphite which has a theoretical capacity of $372 \mathrm{mAh}^{-1} .^{1)}$ As materials which can deliver capacities close to this theoretical value can be easily obtained, it is obvious that next-generation batteries require electrode materials with higher capacities. In recent studies, binary Li alloy materials such as Li-Si or Li-Sn have attracted considerable attention as an alternative negative electrode material. ${ }^{2,3)}$ Especially, silicon based alloys have been regarded to be the most promising alternative to graphite due to its extremely high maximum theoretical capacity of $4200 \mathrm{mAh} \mathrm{g}^{-1}$, which can be achieved when the composition of the alloy reaches $\mathrm{Li}_{4.4} \mathrm{Si}^{4}{ }^{4)}$ However, the practical use of silicon powder as the negative electrode material in Li-ion batteries has not been realized yet, as this material has serious drawbacks; low intrinsic electric conductivity and drastic morphological changes during the insertion/extraction of Li-ions. ${ }^{5,6)}$ Such morphological changes cause the pulverization of the material leading to exfoliation from the electrode. In addition, a breakdown of the conductive network formed between the silicon powder and the current collector occurs. Therefore, even though siliconbased alloys have an extremely high theoretical capacity, their Li-ion capacity fades quite quickly.

To overcome these problems, many researches have attempted to increase the stability of silicon by reducing its size to minimize the effect of volume changes during Li-ion storage, ${ }^{7)}$ or to combine it with active or inactive materials which can act as a matrix to buffer such volume changes. ${ }^{8-11)}$ Combinations of silicon and carbonaceous materials have been studied extensively, but the synthesis methods of such composite materials are generally complicated and additional problems as the formation of inactive $\mathrm{SiC}$ also arise. In addition, although various silicon composite materials have been studied, detailed studies on the morphology of the composite material itself have not been conducted. Spheres are assumed to be a suitable morphology for this purpose, as it can effectively relax internal stresses.

Our strategy to overcome these problems is to encase silicon powder within microspheres of a conductive material which can buffer the volume expansion/shrinkage of the alloy. We chose carbon gels ${ }^{12,13)}$ as the conductive material. Carbon gels are thought to be prospective materials for encasing, as metal powder can be easily dispersed within it, and as it can be easily molded into designated shapes.

Carbon gels are practically aggregates of carbon nanoparticles. The nanoparticles have developed micropores within them, and mesopores are formed between them, so the pore system of carbon gels can be tuned by controlling the growth of the nanoparticles during synthesis, for example, by adjusting synthesis conditions such as the concentration of the catalyst used for synthesis. Therefore, it is assumed that the unique porous structure of carbon gel is favorable for buffering the morphological changes of the alloy. Previously, we verified the validity of this concept. ${ }^{14)}$ In this study, we selected synthesis conditions of the carbon gel matrix, so that the resulting microspheres have a dense surface. The inner part of microspheres synthesized under such conditions also tended to become dense, so the space for buffering was sacrificed, but we thought that the priority was to suppress the formation of solid electrolyte interface (SEI) layers and therefore minimize the irreversible capacity. However, we found that the irreversible capacity caused by the formation of SEI layers was relatively small, and therefore using a carbon gel matrix with a more developed pore system would be more effective for this purpose. 
This work was conducted to clarify how the porous structure of the carbon gel matrix affects the Li insertion/extraction behavior of the carbon gel microspheres encasing silicon powder. First, we encased silicon powder within carbon gel microspheres with a more developed pore system. We reduced the concentration of the carbon source along with the catalyst in the starting solution of the carbon gel matrix. Then the performance of the resulting microspheres was compared with that of microspheres obtained under previous synthesis conditions.

\section{Experimental}

In the preparation of samples, first resorcinol-formaldehyde $(\mathrm{RF})$ solutions were prepared using sodium carbonate $\left(\mathrm{Na}_{2} \mathrm{CO}_{3}\right)(\mathrm{C})$ as the basic catalyst. Commercial $\mathrm{Si}$ powder (Wako Pure Chemical Industries, Ltd., research grade $99.9 \%$ purity) was ballmilled for $24 \mathrm{~h}$ in an argon atmosphere and $\mathrm{Si}$ particles in the range of about 1 to 10 $\mu \mathrm{m}$ were sieved and added to the RF solutions (Fig. 1 (c)). The molar ratio of resorcinol to formaldehyde $(\mathrm{R} / \mathrm{F})$ and the molar ratio of resorcinol to $\mathrm{Si}(\mathrm{R} / \mathrm{Si})$ were respectively fixed to 0.5 and 1.0 . In order to obtain microspheres with a more developed pore structure, the molar ratio of resorcinol to catalyst $(\mathrm{R} / \mathrm{C})$ and the ratio of resorcinol to water $(\mathrm{R} / \mathrm{W})$ were respectively set to $\mathrm{R} / \mathrm{C}=400$ and $\mathrm{R} / \mathrm{W}=0.25 \mathrm{~g} / \mathrm{cm}^{3}$ (sample A). For comparison, microspheres were also synthesized under previously adopted conditions, i.e. $\mathrm{R} / \mathrm{C}=100$ and $\mathrm{R} / \mathrm{W}=0.50$ $\mathrm{g} / \mathrm{cm}^{3}$ (sample B). Next, the solutions were dispersed into cyclohexane containing a surfactant Span 80, and inverse emulsions were formed. The emulsions were stirred until the dispersed solution transformed to gels having a microsphere morphology. The obtained hydrogel microspheres were freeze-dried. Finally, the samples were heated to $1273 \mathrm{~K}$ in a nitrogen atmosphere for $1 \mathrm{~h}$, yielding carbon gel microspheres encasing Si powder.

The surface of the obtained samples was directly

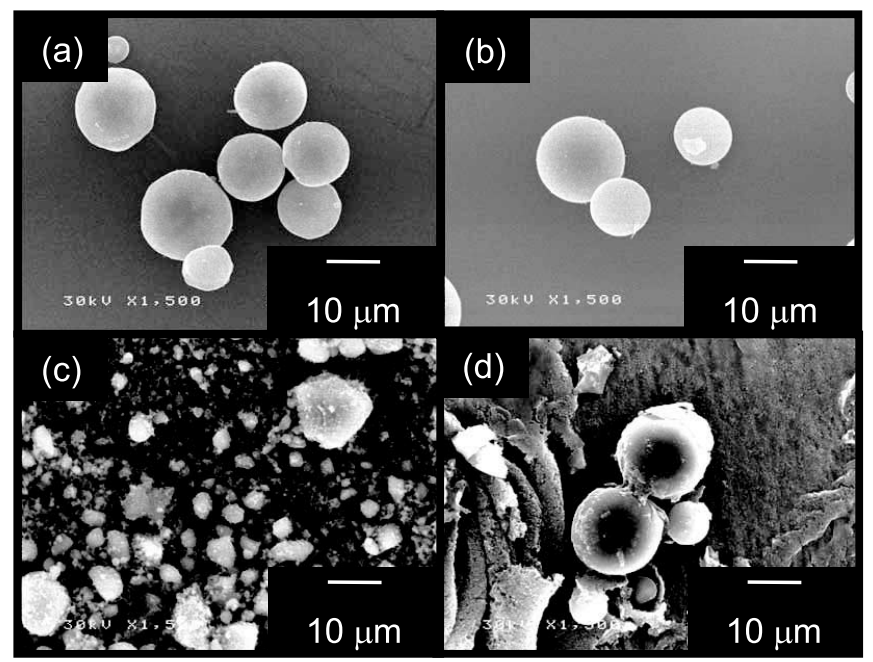

Fig. 1 SEM images of carbon gel microspheres (a) including and (b) not including silicon powder, (c) silicon powder and (d) carbon gel microspheres encasing silicon powder after discharge. observed using a scanning electron microscope (SEM) (JEOL; JSM-5410). The existence and crystallinity of Si in the samples were analyzed by X-ray diffractometry (XRD) (JEOL; JDX-8020) and porous properties were evaluated through nitrogen adsorption experiments conducted using an adsorption apparatus (BEL Japan, Belsorp-mini II). The composition of the samples was evaluated through thermogravimetry (TG) and was found to be $33 \mathrm{wt} . \%$ silicon and $67 \mathrm{wt} . \%$ carbon.

Slurries were prepared by mixing polyvinylidene fluroride, Ketjen black and silicon powder, or carbon gel microspheres encasing and not encasing silicon powder at weight ratios of $5: 40: 55$ or $5: 5: 90$. Charge-discharge properties were evaluated by a battery charge discharge unit HJ1010mSM8A (HOKUTO DENKO) using a Swagelok-type two-electrode cell. Li metal was used as the counter electrode and the electrolyte was a solution of $1 \mathrm{M} \mathrm{LiClO}_{4}$ dissolved in a 1:1 mixture of ethylene carbonate and diethyl carbonate. Celgard ${ }^{\circledR} 2400$ was used as the separator. The cycling rate was fixed to $25 \mathrm{~mA} \cdot[\mathrm{g}$ composite electrode $]^{-1}$, and samples were cycled between 0.02 and $3.00 \mathrm{~V}$ vs. $\mathrm{Li} / \mathrm{Li}^{+}$.

\section{Results and Discussion}

Figure 1 shows SEM images of typical samples obtained in this work. A SEM image of carbon gel microspheres not encasing silicon powder is also shown in Fig. 1 (b) for comparison. Carbon gel microspheres not encasing silicon powder were completely spherical, and those encasing silicon powder were also spherical but were slightly distorted, indicating the inclusion of materials within them. As non-spherical shaped particles such as silicon powder were not observed around the microspheres in these SEM images, it was confirmed that all of the silicon powder was efficiently encased within the carbon gel microspheres.

To identify the existence and to evaluate the crystallinity of the encased silicon powder, XRD analysis was conducted. Figure 2 shows the XRD patterns of carbon gel microspheres including and not including silicon pow-

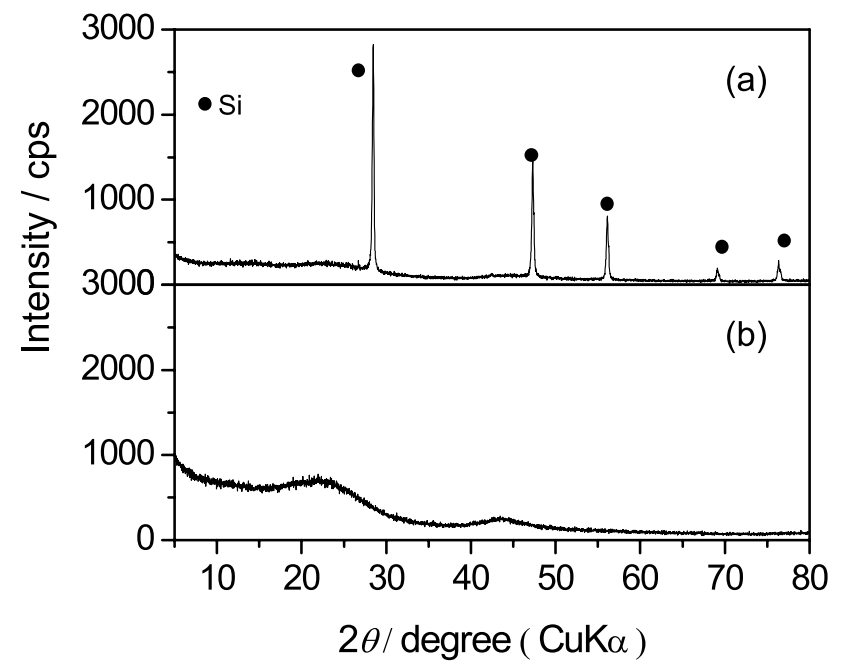

Fig. 2 XRD patterns of carbon gel microspheres (a) encasing and (b) not encasing silicon powder. 
der. In the XRD pattern of carbon gel microspheres without silicon inclusion, only broad peaks can be observed: which are characteristic of amorphous carbon. The XRD pattern of carbon gel microspheres encasing silicon powder included sharp peaks which can be assigned to silicon crystals in addition to the peaks of amorphous carbons, and no other peaks assignable to other materials were observed. The SEM images and results of XRD analysis indicates that silicon powder was successfully encased within carbon gel microspheres without the formation of inactive by-products such as $\mathrm{SiC}$.

Nitrogen adsorption isotherms of silicon powder and carbon gel microspheres encasing silicon powder synthesized are shown in Fig. 3. Nitrogen hardly adsorbed on silicon powders showing that it is a non-porous material. On the other hand, significant adsorption occurred on carbon gel microspheres encasing silicon powder, their isotherms being type I of the IUPAC classification, indicating the development of micro pores within them.

As expected, microspheres synthesized under newly adopted conditions (sample A) had a more developed porous structure than those obtained under previous conditions (sample B). The concentration of the catalyst in the starting solution of sample A was decreased to be much lower than that of sample B. During sol-gel reaction, the catalyst controls the number of the particles which generate in the sol and how the generated sol particles grow. ${ }^{15)}$ Therefore, the nanoparticles which form the matrix of sample A are thought to be larger than those of sample B. As the concentration of resorcinol was also reduced, sample $\mathrm{A}$ has a more developed porous structure and therefore a larger buffering space than sample B. Therefore sample A is expected to have a more effective structure for the relaxation of volume expansion/shrinkage of silicon than sample B.

Figure 4 shows representative charging and discharging curves of silicon powder and carbon gel microspheres encasing or not encasing silicon powder measured at a constant current of $25 \mathrm{~mA} \cdot$ [g-composite elec-

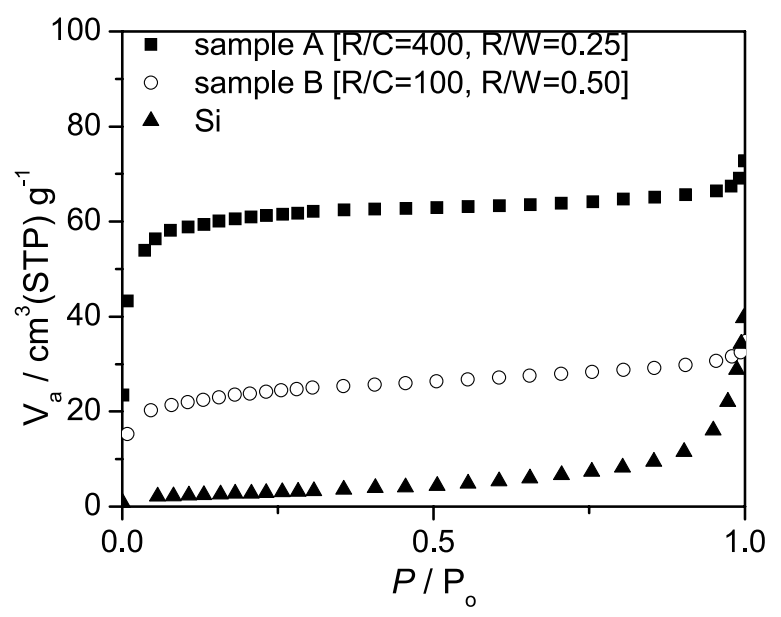

Fig. 3 Nitrogen adsorption isotherms of carbon gel microspheres encasing silicon powder (Sample A and Sample B) and silicon powder. trode $]^{-1}$. During the charging of silicon powder, a single flat plateau at about $0.4 \mathrm{~V}$ was observed which can be ascribed to the de-alloying reaction of LixSi. The chargedischarge curves of carbon gel microspheres not encasing silicon powder showed no plateaus and were typical curves of hard carbon negative electrodes. Carbon gel microspheres encasing silicon powder also showed plateaus at about $0.4 \mathrm{~V}$, and gradual slopes typical of carbon gels. Therefore, it is assumed that the silicon powder encased within carbon gel microspheres is acting as an effective active electrode material and the carbon gel matrix is also contributing to the Li-insertion/extraction capacity. While the silicon electrode showed a drastic decrease in discharge capacity in the second cycle, carbon gel microspheres not encasing silicon powder showed almost a constant discharge capacity. The irreversible capacity of carbon gel microspheres encasing silicon powder was much smaller than that of silicon powder, and the reversible capacity of carbon gel microspheres encasing silicon powder was mostly retained whereas that of silicon powder drastically decreased. The discharge capacity of the second cycle of silicon, sample A, sample B and carbon gel microspheres not encasing silicon were about 420, 720, 610 and 300 $\mathrm{mAhg}^{-1}$ respectively.

The plateau observed at about $0.4 \mathrm{~V}$ in the discharge curve of sample A was longer than that of sample B. As the silicon content of both samples is almost the same, this result indicates that the silicon in sample A can contribute to $\mathrm{Li}$ insertion/extraction more effectively. Moreover, from the shape of the discharge curve, it is assumed that the contribution of the carbon gel matrix to Li insertion/extraction is more significant in sample A. Therefore in carbon gel microspheres encasing silicon powder, it seems to be better to use a carbon gel with a developed porous structure.

Cycle performances of the materials obtained in this work are shown in Fig. 5. The reversible capacity of silicon powder faded quite quickly during cycles, showing its poor cycle stability. During Li insertion/extraction, sil-

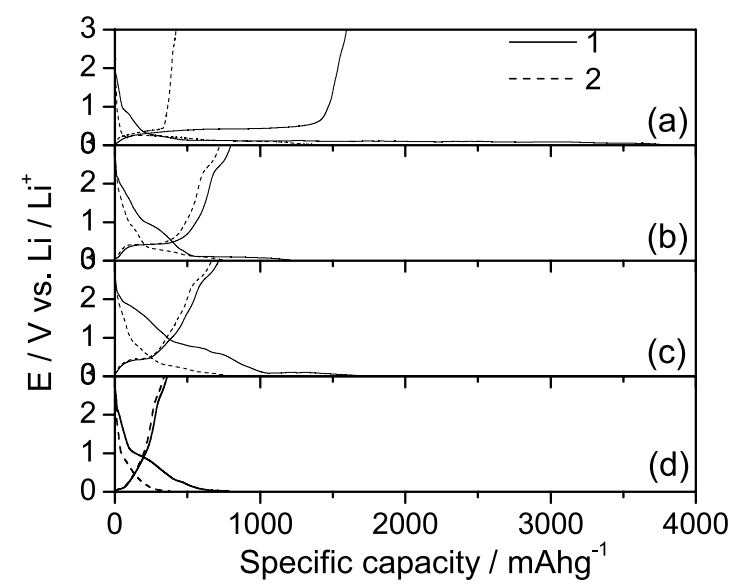

Fig. 4 Representative charge-discharge curves of silicon powder (a), carbon gel microspheres encasing silicon powder (Sample A) (b), (Sample B) (c) and carbon gel microspheres not encasing silicon powder (d). 


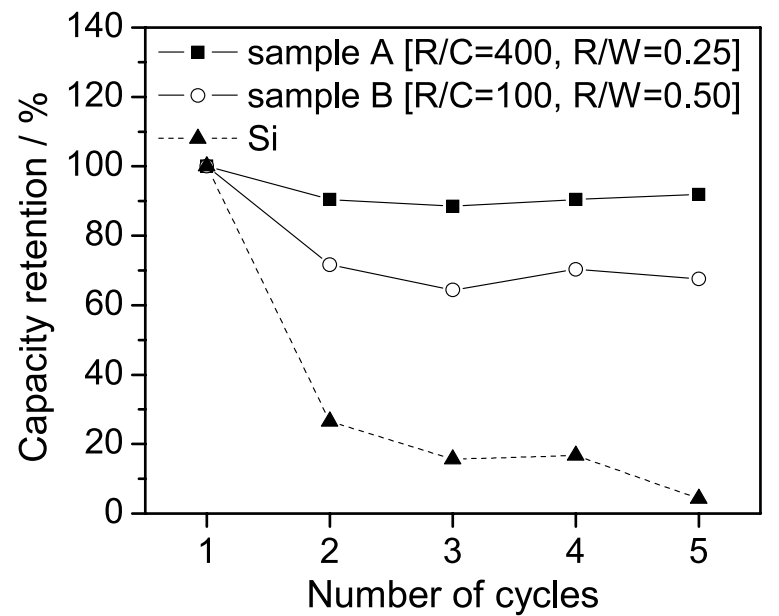

Fig. 5 Cycle behaviors of silicon powder and carbon gel microspheres encasing silicon powder (Sample A and Sample B).

icon experiences a drastic expansion/shrinkage and pulverization of the material occurs leading to severe capacity fading. Compared with silicon powder, carbon gel microspheres encasing silicon powder experienced only a slight decrease in reversible capacity during cycling. This retention of capacity indicates that the morphological change of silicon was relaxed reversibly by the buffering effect of the porous structure of carbon gels. The complete retention of the silicon within the microspheres, even when the silicon particles are pulverized, is also thought to contribute to capacity retention. When the carbon gel microspheres encasing silicon powder are compared, sample A displayed a more excellent cycle performance than sample B. Therefore, developing the porosity of the matrix material is thought to be an effective way to enhance the buffering effect of such materials.

Figure 1(d) shows a SEM image of sample A after charge-discharge tests. The morphology of the microspheres was almost retained but some cracks can be occasionally found. Therefore it is assumed that the performance of carbon gel microspheres encasing silicon powder can be further improved by optimizing material designing parameters such as silicon particle size, the degree of porous structure development and particle size of the microspheres.

\section{Conclusion}

We previously reported the validity of encasing silicon powder within carbon gel microspheres for the development of an anode material for $\mathrm{Li}$ ion batteries. In this work, we focused on the porous structure of the carbon gel matrix which provides the buffering space for the relaxation of volume expansion/shrinkage of silicon during $\mathrm{Li}$ insertion/extraction. It was confirmed that silicon powder encased within carbon gel with a more developed porous structure possesses a higher discharge capacity and shows a better cycle behavior. Therefore, encasing silicon powder within carbon gel microspheres with developed porous structure was proved to be one effective way to bring out the potential of silicon for $\mathrm{Li}$ insertion/extraction. The results obtained in this work also suggested that the performance of such microspheres can be further improved by optimizing material designing parameters such as the porous structure of the carbon gel matrix, silicon particle size and the size of the microspheres.

\section{References}

1) M. Winter, J. O. Besenhard, M. E. Spahr, and P. Novak, Adv. Mater., 10, 725 (1998).

2) U. Kasavajjula, C. S. Wang, and A. J. Appleby, J. Power Sources, 163, 1003 (2007).

3) A. Sivashanmugam, T. P. Kumar, N. G. Renganathan, S. Gopukumar, M. Wohlfahrt-Mehrens, and J. Garche, J. Power Sources, 144, 197 (2005).

4) R. A. Haggins, J. Power Sources, 81, 13 (1999).

5) W. J. Weydanz, M. Wohlfahrt-Mehrens, and R. A. Haggins, J. Power Sources, 81, 237 (1999).

6) H. Li, X. J. Huang, L. Q. Chen, G. Zhou, Z. Zhang, D. Yu, Y. J. Mo, and N. Pei, Solid State Ionics, 135, 181 (2000).

7) H. Li, X. Huang, L. Q. Chen, W. Zhengang, and Y. Liang, Electrochem. Solid State Lett., 2, 547 (1999).

8) N. Dimov, S. Kugino, and M. Yoshio, Electrochim. Acta, 48, 1579 (2003).

9) I. Kim, P. N. Kumta, and G. E. Blomgren, Electrochem. Solid State Lett., 3, 493 (2000).

10) G. X. Wang, L. Sun, D. H. Bradhurt, S. Zhong, S. X. Dou, and H. K. Liu, J. Power Sources, 88, 278 (2000).

11) M. D. Fleischauer, J. M. Topple, and J. R. Dahn, Electrochem. Solid State Lett., 8, A137 (2005).

12) R. W. Pekala, J. Mater. Sci., 24, 3221 (1989).

13) R. W. Pekala and D. W. Schaefer, Macromoleculues, 26, 5487 (1993).

14) T. Hasegawa, S. R. Mukai, Y. Shirato, and H. Tamon, Carbon, 42, 2573 (2004).

15) Y. Yamamoto, T. Nishimura, T. Suzuki, and H. Tamon, J. Non-cryst. Solids., 288, 46 (2001). 